International Journal of Canadian Studies

Revue internationale d'études canadiennes
INTERNATIONAL JOURNAL OF CANADIAN STUDIES

REVUE INTERNATIONALF D'ÉTUDES CANADIENNES

\title{
Une politique du bien commun au Canada est non seulement possible, mais nécessaire
}

\section{Charles Blattberg}

Numéro 44, 2011

URI : https://id.erudit.org/iderudit/1010088ar

DOI : https://doi.org/10.7202/1010088ar

Aller au sommaire du numéro

Éditeur(s)

Conseil international d'études canadiennes

ISSN

1180-3991 (imprimé)

1923-5291 (numérique)

Découvrir la revue

Citer cet article

Blattberg, C. (2011). Une politique du bien commun au Canada est non seulement possible, mais nécessaire. International Journal of Canadian Studies / Revue internationale d'études canadiennes, (44), 203-212.

https://doi.org/10.7202/1010088ar 


\section{Charles Blattberg}

\section{Une politique du bien commun au Canada est non seulement possible, mais nécessaire}

« Est-ce que je ne détruis pas mes ennemis quand je les transforme en amis? » Abraham Lincoln (Dancă 65, traduction libre)

À la fin de son texte « Une politique du bien commun au Canada est-elle possible? », Stéphane Courtois avance la critique suivante au sujet de ma philosophie politique " patriotique »: devant les défis du pluralisme dans les sociétés contemporaines, l'affirmation d'un idéal qui suppose que les citoyens dans un pays comme le Canada puissent affirmer une conception substantielle de leur bien commun (une conception qui représenterait, entre autres, un rapprochement des différentes communautés nationales dans le pays), n'est-elle pas au bout du compte irréaliste, voire utopique? (Courtois, 2010, 281) Bien sûr, je n'ai jamais caché ma conviction selon laquelle le bien commun ne peut être développé que par le mode du dialogue extrêmement fragile qu'est la conversation, et non par celui, plutôt antagoniste, qu'est la négociation. Cela parce que même si la négociation permet de trouver des accommodements raisonnables aux conflits, seule la conversation peut mener à de véritables rapprochements. Mais qu'en est-il de la conversation en politique? Étant donné l'immense disparité de pouvoir qui existe entre les citoyens, mon manque de réalisme s'aggraverait d'une bonne dose de naïveté.

En fait, ces deux critiques sur l'utopie et la naïveté tendent à venir de disciplines différentes : c'est de philosophes comme Courtois que vient la suggestion que mon approche est utopique, tandis que les politologues, comme Éric Montpetit et Alain Noël, la trouvent naïve. Ainsi, Montpetit écrit que l'approche patriotique vise à remplacer la vie politique « normale », qui relève du pouvoir, par " l'activité intellectuelle » qui se réduit à l'affirmation de « visions » dans la conversation (Montpetit, 2008, 21; traduction libre), alors que Noël classe le patriotisme parmi les approches qui, « d'une manière plutôt abstraite ", mettent trop l'accent sur les principes et pas assez sur le pouvoir (Noël, 2008, 428, 432; traduction libre). Michel Seymour se joint quant à lui aux deux critiques. Il relève la naïveté, parce que le patriotisme échoue à voir que « dans la réalité concrète des rapports de domination politiques », on passe de la force à la négociation, puis à la conversation « et non l'inverse ». Il souligne aussi l'utopie liée à ma croyance en l'existence d'une « illusoire communauté politique » et à ma conception de la communauté nationale qui va à l'encontre de l'immense majorité des gens (y compris mes camarades les Canadiens anglais) - qui, je dois le présumer, souffrent d'une forme de « fausse conscience » (Seymour, 2008, 125, 123, 257). En somme, on pourrait dire que je rêve de l'impossible : de concitoyens qui feraient preuve d'une générosité jusqu'ici inconnue lorsque des conflits de fond portent sur des 
valeurs jugées primordiales. Voilà un idéal aimable,, même touchant, mais totalement irréaliste.

Il y a pourtant ici une grande ironie. J'estime en effet que c'est mon approche qui est la plus réaliste et que l'incapacité à le percevoir est elle-même le résultat de l'utopie et de la naïveté. Le réalisme du patriotisme est fondé sur l'idée qu'il est souvent dans l'intérêt des gens de converser. Non parce que la conversation suppose une doctrine smithienne de « l'intérêt personnel éclairé » selon laquelle, grâce à une main invisible, on promeut les intérêts de la société à travers l'avancement de ses propres intérêts (Smith, 1991, livre 4, chapitre 2, paragraphe 9). Le patriotisme ne suppose pas non plus une doctrine tocquevillienne de « l'intérêt bien entendu » qui suggère qu'il est, en fin de compte, dans l'intérêt personnel de chaque membre d'une communauté de faire des sacrifices limités au nom du bien de tous (Tocqueville, 1961, partie 2, chapitre 8). La réconciliation des valeurs réalisée par une conversation réussie n'est pas accomplie par une raison instrumentale, comme chez Smith, et son résultat ne doit pas être conçu comme imposant des sacrifices, comme chez Tocqueville, parce que la raison pratique en question ici est transformationnelle : c'est en transformant nos interprétations, et donc le sens de nos valeurs qu'on réussit par une conversation à les rapprocher, et qu'il en résulte des interprétations qui nous semblent mieux réaliser l'esprit de nos valeurs que lorsque le conflit a commencé. C'est donc grâce à l'apprentissage qu'on peut parler de réconciliations et de compréhensions partagées n'exigeant aucun compromis. De tels compromis sont par contre inéluctables lorsqu'il est question d'accommodements obtenus par la négociation.

J'essaierai donc, en réponse aux allégations d'utopisme, d'expliquer pourquoi il est dans l'intérêt des plus forts de s'engager dans une conversation ainsi comprise et, de plus, pourquoi c'est la notion des philosophes posant que nous devrions viser un bien commun beaucoup moins substantiel, sinon totalement procédural, un bien commun représenté par une théorie de la justice, qui est utopique. Puis, en réponse à l'argument de naïveté, je montrerai pourquoi c'est précisément en raison des disparités de pouvoir entre les personnes qui existent dans un pays comme le Canada qu'il est dans l'intérêt des faibles de s'engager dans la conversation, et pourquoi ce sont ceux qui affirment le contraire qui succombent à la naïveté.

\section{Conversation pour les forts}

Que leur conception se traduise par des règles sur la justice distributive ou sur la meilleure manière de délibérer, les philosophes qui posent que le bien commun d'une société passe par une théorie définie comme un ensemble de principes systématiquement imbriqués, que tous les citoyens devraient accepter, ne fontils pas preuve d'une forme moniste d'utopisme? Car même dans une version minimaliste, il reste peu réaliste de croire que les valeurs exprimées par ces 
principes puissent être assemblées sans distorsion, ou même sans mutilation. Cette idée va à l'encontre du fait souvent souligné par les penseurs pluralistes, à savoir que les valeurs tendent à être incommensurables les unes par rapport aux autres. Les pluralistes vont toutefois trop loin lorsqu'ils estiment qu'il n'existe pas de bien commun, qu'on ne peut jamais espérer l'intégration ou la réconciliation des valeurs qui font partie d'un tout. Néanmoins, nous devons accepter qu'il y aura toujours des conflits fondamentaux sur le sens de ce bien commun; il serait utopiste de croire le contraire.

L'autre aspect utopique de la pensée des théoriciens de la justice relève de leur croyance en la possibilité d'un consensus, où tout le monde serait d'accord pour se soumettre aux règles d'un tel « jeu ». Selon John Rawls :

Tout comme des joueurs ont pour but commun de bien jouer et de façon correcte (fair), de même les membres d'une société bien ordonnée ont pour but commun de coopérer afin de réaliser leur propre nature d'une manière qu'autorisent les principes de la justice (Rawls, Théorie de la justice, 570; voir aussi Rawls, Libéralisme politique, 249).

Dans une telle perspective, il me semble qu'une attitude est présupposée chez les « joueurs » potentiels, attitude qui, surtout en ce qui concerne ceux qui sont forts, n'a tout simplement aucun sens. En effet, pourquoi quelqu'un qui se prend lui-même au sérieux (ainsi que ses préoccupations) serait-il prêt à respecter, plutôt que de donner du bout des lèvres, les restrictions que ces règles impliquent (Blattberg, «Taking Politics Seriously »)? De ce point de vue, il est inutile d'arguer, comme des théoriciens tels que Mark Kingwell ont fait au Canada, que notre politique devrait redevenir plus civile (Kingwell, Civil Tongue; "Shout Doctrine »; " "Fuck You" »). Cette critique laisse entendre que la civilité est une compétence sociale et que cette compétence devrait être un élément immuable de nos régimes politiques . Mon intention ici n'est pas de suggérer que nous ne devrions pas être courtois, mais plutôt que la préoccupation pour la civilité révèle une ignorance des exigences du meilleur des dialogues. Une conversation ne peut pas être habile, car elle vise, comme je l'ai suggéré, à transformer le sens, souvent de manière profonde. C'est pour cette raison que les conversations ont tendance à être si douloureusement maladroites : les interlocuteurs empruntent un chemin risqué, car véritablement inconnu, puisqu'il ne peut pas être balisé par des idées ou des règles préexistantes. Cela, comme l'ont souligné des psychologues, n'est pas du tout amusant, car le plaisir intellectuel et le confort existentiel ne viennent pas du réexamen des préjugés intéressés, mais du raffinement des idées selon des formations déjà maîtrisées (Nickerson, 1998). La conversation, pourrions-nous dire, engage ainsi une forme de raison pratique qui est plus proche de l'esprit de la phronèsis (la sagesse pratique) d'Aristote que de sa technè (bien qu'il faille reconnaître que, étant donné que sa conception de la première est fondée sur la théorie, elle est aussi trop technique). 
Par ailleurs, nous avons à nous interroger sur la sagesse de certaines de ces règles. Prenons le principe cher aux théoriciens de la démocratie délibérative, voulant que les interlocuteurs doivent être égaux. Pourquoi une telle chose serait-elle nécessaire, étant donné les innombrables conversations fructueuses qui ont bien évidemment lieu entre les parents et leurs enfants, les enseignants et leurs élèves, ou les patrons et leurs employés? Cela me dépasse. Et même si on fait abstraction des différences de pouvoir, d'argent ou de position sociale entre les gens, il restera, la plupart du temps, une différence dans l'intelligence et il est certain que la délibération efficace ne peut exiger l'abandon de cette qualité. Il me semble alors que Martin Buber a raison quand il souligne la nécessité, non pas que les interlocuteurs soient égaux, mais seulement qu'il y ait une « symétrie » entre eux, celle qui survient lorsque chacun est disposé à parler et à vraiment écouter à son tour (Buber, 1959; voir aussi Blattberg, " Démocratie patriotique »). Ainsi, la revendication de Rawls selon laquelle une société régie par une théorie de la justice soit une « utopie réaliste » est tout simplement un oxymoron qui doit être rejeté.

Pourtant, à l'idée d'abandonner la perspective théorique, les théoriciens craignent ce qu'ils croient être la seule alternative : la realpolitik. Or en régime de realpolitik, parler des valeurs comme si elles représentaient des vérités morales ne fait que cacher la réalité puisque, comme Hobbes l'a dit, " quel que soit l'objet de l'appétit ou du désir que l'on éprouve, c'est cet objet qu'on appelle bon; et l'objet de notre haine et de notre aversion est ce qu'on appelle mauvais; l'objet de notre mépris, on le dit abject et méprisable » (Hobbes, 2000, partie I, chapitre 6). Mais le fait est que personne, sauf le nihiliste, n'utilise ces mots d'une manière si désinvolte. Les gens ne peuvent dire ni croire n'importe quoi, parce que la moralité est réelle; voilà pourquoi on ne peut s'empêcher de faire des justifications morales. La croyance selon laquelle les intérêts ne comprennent pas de valeurs est donc incohérente. Mieux vaut parler, avec Bernard Crick, des intérêts « moraux ou matériels » (Crick, 1992, 163; traduction libre).

Alors, il se trouve que parmi les valeurs partagées par la plupart d'entre nous, il y a la vertu de la tolérance. C'est pourquoi il est dans l'intérêt, même des forts, de négocier leurs conflits de bonne foi. Étant donné qu'on ne veut pas écraser un concitoyen avec qui on se trouve en désaccord, on croit qu'il faut faire des concessions non seulement parce que l'on se sent sous pression, mais aussi parce que l'autre mérite un minimum de respect. Certes, on ne partage pas les mêmes interprétations des valeurs avec lui, mais il demeure un être moral (ce n'est pas sa faute s'il a été élevé différemment). Bien sûr, il y a ici des limites : nous devons nous demander à quel degré notre mode de vie est menacé et combien de terrain nous pouvons céder. Mais, quand même, on est prêt à tolérer les modes de vie des autres jusqu'à un certain point. Partant, on peut envisager un accommodement avec eux. Cela fait partie de notre mode de vie. 
Mais pourquoi ne pas viser plus haut? Après tout, personne, encore moins les forts, n'aime faire des concessions. Et si on veut les éviter, il est dans notre intérêt d'essayer la conversation, de s'efforcer de rapprocher les positions plutôt que de lutter pour des accommodements. En fait, grâce à la conversation, il est non seulement possible d'éviter les concessions, mais aussi d'apprendre comment rester plus fidèles à nos valeurs. Étant donné ce que l'on entend, si on écoute bien, lors d'une recherche du bien commun, il est possible de développer une meilleure interprétation de ses valeurs. Après tout, personne, peut-être surtout les forts, ne possède une interprétation parfaite de ses propres valeurs - il y a toujours plus à apprendre sur soi.

\section{Conversation pour les faibles}

On peut résumer - et cela a été fait - la critique de la naïveté à mon endroit en une phrase : je rêverais de faire du monde politique un séminaire de philosophie. Mais ayant moi-même fait partie de plus d'un tel séminaire, même ce contexte ne me semble pas suffisamment propice à la conversation. Car, bien qu'il le fasse avec exagération, Robert Musil touche à une vérité lorsqu'il affirme que « les philosophes sont des violents qui, faute d'armée à leur disposition, se soumettent le monde en l'enfermant dans un système » (Musil, 1956, 319). Cela ne s'applique pas seulement à certains théoriciens philosophes, mais aussi à leurs némésis, les philosophes de la différence selon qui « pour supprimer la violence, il faut recourir à la violence » (Levinas, 1994, 75) et donc déconstruire la théorie, « violence contre violence » (Derrida, 1967, 172). Il devrait être évident que ces individus ne feraient pas de bons partenaires de conversation. Bien sûr, j'évoque ici une longue histoire : le démantèlement par Socrate de ses interlocuteurs dans les soi-disant « dialogues » de Platon; l'incapacité de Boèce à recevoir une oreille attentive de Philosophie, son « infirmière » avec la bouche pleine de métaphores martiales; la volonté des maîtres chez Hegel de lutter jusqu'à la mort avec leurs esclaves pour la reconnaissance; l'affirmation de John Stuart Mill selon laquelle la vérité est issue « le plus correctement possible, c'est-à-dire brutalement, par une lutte entre des combattants enrôlés sous des bannières opposées »; et enfin même les éloges d'Habermas sur les échanges dans lesquels « aucune contrainte ne s'exerce en dehors de celle du meilleur argument », l'association de l'argumentation avec la force dans la philosophie est endémique (Mill, 1990, 133; Habermas, 1978, 149; Blattberg, « Critique de l'interprétation »; Blattberg, « Exiger la reconnaissance? »). Peut-être cette histoire aboutit-elle à la récente affirmation autodestructrice voulant que la raison n'ait pas évolué pour atteindre la vérité, mais plutôt afin de gagner des arguments (Mercier et Sperber, 2011).

En somme, si jamais la conversation doit prendre une place importante dans notre vie politique, il sera nécessaire de surmonter les présupposés sur le dialogue que nous avons hérités de certains philosophes et qui ont fini par être intégrés au sens commun. Cet héritage me semble expliquer pourquoi, quand 
nous sommes impliqués dans un conflit politique, nous avons tendance non à répondre comme un opposant qui n'est pas un adversaire, ou à écouter avec un esprit ouvert dans l'espoir de développer une réconciliation, mais plutôt à nous battre pour défendre une position formulée antérieurement. C'est le débat et non la conversation qui l'emporte. Ainsi, dans la section intitulée « Un débat public nécessaire » de son livre La voie canadienne, le théoricien Will Kymlicka établit clairement que son argument revient à préciser l'existence de « certaines conditions non négociables » de la citoyenneté canadienne (Kymlicka, 2003, 111). Invoquant le débat sur le hijab dans les écoles du Québec, Kymlicka loue son résultat final : le dépassement du stéréotype selon lequel les musulmans n'acceptent pas ces exigences. Parce que les Québécois, écrit Kymlicka :

avaient des objections légitimes à opposer à certaines pratiques, lesquelles méritent justement d'être combattues. Avant que les Québécois ne soient en mesure d'accepter le hijab dans les écoles et ne souhaitent l'y accueillir (" to accommodate ») donc, on devait les convaincre qu'une telle décision ne signifierait pas forcément qu'ils approuvaient les autres pratiques traditionnelles. Ils étaient prêts à appuyer une certaine prise en compte de la diversité, mais seulement après qu'on les eut convaincus qu'il existait des limites au processus présidant à une telle prise en compte (Kymlicka, 2003, 112).

Je suis tout à fait pour l'élimination des stéréotypes, mais expliciter les limites préétablies me semble un résultat loin d'être adéquat. En tant que père d'une petite fille, je me trouve de plus en plus sensible à - pour mieux dire, choqué par - la sexualisation croissante des filles toujours plus jeunes dans notre société, que ce soit dans les médias ou dans nos rues. Alors que le hijab n'est pas, bien sûr, la solution pour ceux d'entre nous qui ne sont pas musulmans (ni même, il va sans dire, pour tous les musulmans), il est clair que nous avons tous manqué une occasion de nous engager dans une discussion bien nécessaire au sujet du sens de la modestie. Et une telle discussion n'en est pas seulement une à partir de laquelle les membres de la communauté musulmane ont quelque chose à apprendre; ils ont aussi clairement quelque chose à y apporter. Pourtant, la seule question posée sur de tels sujets est toujours « dans quelle mesure faut-il accommoder? », jamais « pourquoi vous engagez-vous dans cette pratique? » ni « avez-vous des raisons pour cela que vous êtes disposé à partager, ainsi que peut-être à changer? » (Blattberg, «L'Esprit d'Al-Andalous »).

Quand tout le monde, y compris les philosophes, réclame plus de débats, il est évident que les gens les moins aptes à faire avancer leurs causes dans des contextes hostiles vont perdre. C'est ce qui explique, me semble-t-il, la situation terrible dans laquelle les autochtones du Canada se trouvent encore aujourd'hui (Roy, 2010). Les faibles ont tendance à être désavantagés quand il s'agit de lutter avec les forts, justement parce qu'ils sont faibles. C'est l'incapacité à constater ce fait qui est une marque de naïveté. Ainsi, au lieu 
de dire aux faibles de se battre pour la justice, nous devrions travailler pour une société dans laquelle ils peuvent converser, dans laquelle ils peuvent non vaincre leurs opposants, mais changer leurs idées. Or, même si cet objectif est dans l'intérêt de tous, nous n'avons pas fait assez pour le rendre possible. Je n'ai jamais prétendu qu'il existe une garantie en vertu de laquelle la conversation pourrait réconcilier tous, ou même la plupart de nos conflits; cela dit, il y a bel et bien une garantie que nous n'atteindrons jamais la réconciliation dans notre vie politique sans elle. Et pourtant, nous ne lui avons jamais donné une vraie chance.

\section{Conclusion}

Seymour demande : «Ai-je tort d'affirmer que, lorsqu'elle est appliquée dans le contexte des relations Québec-Canada, la position de Blattberg équivaut à demander au Québec de reconnaître la nation canadienne avant de commencer le dialogue portant sur une hypothétique reconnaissance du peuple québécois? »(Seymour, 2008, 124). Oui, il a tort, et pas seulement parce que je conçois le Canada comme une communauté politique et non nationale. Mais je dois admettre que la suggestion de Seymour voulant que mon point de vue « revien[ne] à dire que le Québec devrait renoncer définitivement à la menace de la sécession comme moyen de pression, sous prétexte que cela ressemble trop à une négociation » (Seymour, 2008, 124) vient comme une sorte de soulagement. Après des années à avoir été considéré comme naïf, il est bon de voir qu'on reproche à ma philosophie politique de n'être qu'un stratagème rhétorique conçu pour retirer aux Québécois leur droit de brandir cette menace. Cela dit, la maxime pratique que je propose, d'abord la conversation, puis la négociation, puis la force, est destinée uniquement à ceux qui souhaitent répondre aux conflits qui surgissent entre eux et leurs compatriotes - ceux avec lesquels ils partagent et souhaitent continuer à partager un bien commun politique. S'ils ne le désirent pas, ce qui serait légitime bien que regrettable, alors à ceux-ci, comme je l'ai souligné dans mon livre Et si nous dansions? Pour une politique du bien commun au Canada, « nous n'avons pas grand-chose à dire » (Blattberg, 2004, 144). Le patriotisme comme je le conçois comprend les relations entre les citoyens comme quelque chose qui s'apparente à une forme d'amitié aristotélicienne. Il devrait donc être évident que si quelqu'un ne souhaite plus partager l'amitié (ou s'il ne l'a jamais souhaité), aucun argument, philosophique ou autre, n'aura d'effet (positif). Ce qu'il faut à la place, je l'ai dit ailleurs, c'est quelque chose de beaucoup plus proche de « la séduction » (Blattberg, 2004, 149).

Je ne veux pas dire que la position de Seymour se réduit à rejeter l'idée selon laquelle le Canada pourrait être un pays qui rend justice à sa nature multinationale. Seymour ne fait pas partie des souverainistes « purs et durs. » De ce fait, je crois que le patriotisme a quelque chose à lui dire. Commençons par l'exemple d'un couple marié qui éprouve un conflit profond. La femme 
croit que l'homme a tort de penser qu'il peut aller avec d'autres femmes, que la monogamie n'est pas une base essentielle au sens de leur mariage. Dans ce cas, je dirais que c'est dans l'intérêt des deux d'aborder leur conflit, au départ, par la conversation. Cela veut dire qu'ils vont discuter sur le sens du bien commun qu'est leur mariage. Ainsi, ils peuvent s'assoir autour de la table de cuisine et parler face à face. Ce qu'il faut sur leur mur en arrière-fond, c'est une plaque sur laquelle est écrit « notre mariage. »C'est le sujet de la discussion et, pour l'aborder correctement, personne ne doit mettre en question l'existence de ce bien commun. Autrement dit, personne ne doit mentionner le mot « divorce. » Bien sûr, il est possible et légitime que, s'ils ne peuvent pas régler leur conflit, il y ait en bout de piste un divorce. Mais la conversation est un mode de dialogue trop fragile pour survivre si cette idée est explicitement mise sur la table.

Revenons au cas du Canada. Pour moi, le mariage représente le Canada (même si, bien sûr, l'analogie a ses limites). Je crois que s'il y a deux représentants qui discutent, il faut que l'un représente les Franco-Québécois et l'autre, les autres Canadiens. Cela signifie que, sur le mur en arrière-fond, il devrait y avoir une plaque sur laquelle on voit le drapeau canadien. Il ne faut pas un représentant du Canada face à un représentant du Québec, mais deux Canadiens, et le sujet de leur discussion, ce qui est en question, c'est « Qu'est-ce que c'est le Canada? » ou « Quel est le sens du bien commun canadien? », ou encore « Est-ce un pays comprenant une nation franco-québécoise ou non? ». Il ne s'agit donc pas de la reconnaissance (ou non) de la nation après la reconnaissance du Canada, mais de la reconnaissance (ou non) de la nation avec une certaine reconnaissance du Canada. Laquelle? Il faut en discuter. Et dans une telle discussion, personne ne doit faire allusion à un « couteau sur la gorge » (Léon Dion), ou à un « ultimatum » (Charles Taylor), ni même se présenter comme un être déjà séparé du Canada qui veut négocier de bonne foi (Seymour), position presque aussi menaçante que les deux autres.

Voilà ce que je crois être le meilleur chemin vers un Canada véritablement multinational. C'est une fin essentielle étant donné que le monde, selon certains anthropologues, contient peut-être des milliers de communautés nationales et qu'on risquerait la catastrophe si chacune réclamait son propre État, avec un siège à côté des 194 États siégeant actuellement à l'Assemblée générale de l'Organisation de Nations Unies (institution cruellement mal nommée). L'idée selon laquelle de nombreuses nations pourraient, non seulement « malgré », mais aussi « à cause » de leurs différences, partager le même État doit donc se démarquer autant que possible du modèle décrépi et taché de sang de l'« Étatnation » hérité des traités de Westphalie. Et si nous Canadiens, héritiers d'une histoire relativement paisible, ne pouvons gérer cela, alors qui le peut?

Il y a des tempêtes qui s'annoncent à l'horizon - pas seulement environnementales, mais aussi économiques et d'ordre terroriste - avec leur lot de conflits 
profonds. Espérer les résoudre en ne misant que sur des impositions judiciaires basées sur des règles théoriques ou même des accommodements négociés de bonne foi signifierait produire des lois qui seraient toujours insatisfaisantes, parce qu'aliénantes aux yeux des citoyens. À cause de la fragilité de ces lois, il me semble irréaliste d'émettre l'hypothèse selon laquelle ces dernières et la vie politique « normale » qui les a formulées seraient acceptables. Partant, si nous voulons éviter notre propre « moment de Weimar» dans ce pays, nous avons besoin d'une démocratie beaucoup plus durable que celle que nous avons aujourd'hui. Nous devons tout simplement faire mieux.

\section{Note}

L'auteur remercie Éric Montpetit, Alain Noël et Michel Seymour d'avoir fourni des commentaires sur une version antérieure de ce texte.

\section{Bibliographie}

Blattberg, Charles. Et si nous dansions? Pour une politique du bien commun au Canada, Montréal : Presses de l’Université de Montréal, 2004. . " La démocratie patriotique, pas délibérative », trad. Magdelena Dembinska et Irene Salenson, Éthique publique 7.1 (printemps 2005) : 154-165. . « Exiger la reconnaissance? », trad. Roseline Lemire-Cadieux. La reconnaissance dans tous ses états. Éd. Michel Seymour. Montréal : Québec Amérique, 2009, p. 69-101.

. «Critique de l'interprétation gadamériene de Platon », trad. Monica Émond. Les usages des anciens dans la pensée politique contemporaine. Éds. Martin Breaugh et Yves Couture. Québec : Presses de l’Université Laval, 2010, p. 237-252. . "L'Esprit d'Al-Andalous et la philosophie du dialogue », Arabitudes :

L'Altérité Arabe au Québec, Éd. Henda Ben Salah. Montréal : Fides, 2010, p. 63-67.

. "Taking Politics Seriously - but Not Too Seriously". En ligne : http://papers. ssrn.com/sol3/papers. cfm?abstract_id $=1723387$.

Buber, Martin. « Dialogue ». La vie en dialogue, trad. Jean Loewenson-Lavi. Paris : Aubier, 1959, p. 105-147.

Courtois, Stéphane, «Une politique du bien commun au Canada est-elle possible? », Revue internationale d'études canadiennes 42 (2010) : 273-282.

Crick, Bernard. In Defense of Politics, 4 ed. London: Weidenfeld \& Nicolson, 1992.

Dancă, William (Éd.). Truth and Morality: The Role of Truth in Public Life.

Washington: Council for Research and Values in Philosophy, 2008.

Derrida, Jacques. "Violence et métaphysique: Essai sur la pensée d'Emmanuel

Levinas", L'écriture et la différence. Paris : Seuil, 1967, p. 117-228.

Habermas, Jürgen. Raison et légitimité : Problèmes de légitimation dans le capitalisme avancé, trad. Jean Lacoste. Paris : Payot, 1978.

Hobbes, Thomas. Léviathan, ou, Matière, forme et puissance de l'État chrétien et civil, trad. Gérard Mairet. Paris : Gallimard, 2000.

Kingwell, Mark. A Civil Tongue: Justice, Dialogue, and the Politics of Pluralism, University Park, PN: Pennsylvannia State University Press, 1995. ."The Shout Doctrine" The Walrus (avril 2010). 
." "Fuck You' and Other Salutations: Incivility as A Collective Action Problem", Civility in Politics and Education. Éds. Deborah Mower et Wade L. Robison. New York: Routledge, 2011.

Kymlicka, Will. La voie canadienne : repenser le multiculturalisme, trad. Antoine Robitaille. Montréal : Boréal, 2003.

Levinas, Emmanuel. "Transcendance et hauteur", Liberté et commandement. Paris : Fata Morgana, 1994.

Mercier, Hugo et Dan Sperber. "Why Do Humans Reason? Arguments for an Argumentative Theory", Behavioral and Brain Sciences 34.2 (2011) : 57-74.

Mill, John Stuart. De la liberté, trad. Laurence Lenglet et Dupond White. Paris : Gallimard, 1990.

Montpetit, Éric. "Easing Dissatisfaction with Canadian Federalism? The Promise of Disjointed Incrementalism", Canadian Political Science Review 2.3 (décembre 2008) : 12-28.

Musil, Robert. L'homme sans qualités, tome 1, trad. Philippe Jaccottet. Paris : Seuil, 1956.

Nickerson, Raymond S. "Confirmation Bias: A Ubiquitous Phenomenon in Many Guises”, Review of General Psychology 2.2 (juin 1998) : 175-220.

Noël, Alain. "Democratic Deliberation in a Multinational Federation", Critical Review of International Social and Political Philosophy 9.3 (septembre 2008) : 419-444.

Rawls, John. Libéralisme politique, trad. Catherine Audard. Paris : Presses universitaires de France, 1995.

. Théorie de la justice, trad. Catherine Audard. Paris : Seuil, 1997.

Roy, Jean-Olivier. Les nations autochtones et non-autochtones au Canada: Vers une relation postcoloniale?. Saarbrücken : Éditions universitaires européennes, 2010.

Seymour, Michel. De la tolérance à la reconnaissance : Une théorie libérale des droits collectifs. Montréal : Boréal, 2008.

Smith, Adam. Recherches sur la nature et les causes de la richesse des nations, trad. Germain Garnier et Adolphe Blanqui. Paris : Flammarion, 1991.

Tocqueville (de), Alexis. De la démocratie en Amérique, tome 2. Paris : Gallimard, 1961. 